\title{
Fractal Manifold Method in Systems with Self-Organized Criticality
}

\author{
V.V. Vladimirov ${ }^{*}$, E.V. Vladimirova ${ }^{2}$ \\ ${ }^{1}$ Irkutsk State University, Irkutsk, Russia. \\ ${ }^{2}$ Lomonosov Moscow State University, Moscow, Russia.
}

\begin{abstract}
Complex nonlinear dynamic systems with a tendency to selforganization are considered herein. The article is aimed at obtaining a quantitative assessment of self-organization criticality in theoretical and practical aspects for computer calculations. The problem of direct processing of large arrays of non-Gaussian data is solved without the usual prior transformation into Gaussian data. The proposed method is relevant for situations when the cross-correlation of data in the array is significant, reflecting such processes as avalanches, chain reactions, and collective effects. In the simplest case of one-dimensional space, an algorithm is given for construction of a fractal manifold - a new mathematical object, the argument for which was proposed in [9]. The construction of the fractal manifold made it possible to reveal an unusual property of the Gauss function, which confirms the chosen approach. The fractal manifold method makes it possible to determine more accurately the average value due to its smaller scale in comparison to the Euclidean scale. The algorithm is invariant for linear transformations of the initial data set, has renormalization-group invariance, and determines the intensity of cross-correlation (self-organized criticality effect) of the data. The description of the self-organized criticality state is universal and does not depend on the nature of data correlation similar to the universality of the random variables' distribution in the absence of data correlation. This method can be used with large sets of non-Gaussian or strange data obtained in information technology.
\end{abstract}

Keywords: self-organized criticality, fractal manifold, scale invariance, avalanche, chain reaction, collective effect, the Gauss function, the Bessel function.

\section{INTRODUCTION}

The concept of self-organized criticality $[1,2]$ is recognized as a generalization with a paradigmatic meaning as a form of generalization that will characterize the next stage of physics [3]. The concept has provided a consolidation for the phenomena of a wide range of disciplines, covering solar physics, astrophysics, magnetospheric physics, geophysics, biophysics, and social sciences, which had no context [4]. The processes of self-organized criticality (SOC) are observed in some complex systems consisting of many components, interacting in the near and far order, for example, in neural networks, forest fires, and electrical networks that form avalanches, chain reactions, and collective effects.
For example, according to experimental data, the brain of healthy mammals functions in a state of SOC. When brain function is impaired during epileptic seizures, the neural network loses its criticality characteristics [5]. Artificial neural networks do not simulate the process of SOC, which can be assessed as a problem requiring a solution [6].

SOC systems are characterized by scale invariance and criticality achievement without control parameters. The fractals are of great interest due to their scale invariance, and selfsimilar geometry of fractal objects. However, the question "Fractals: Where's the Physics?" remains relevant [7]. Moreover, a criticality indicator is also required to compare quantitatively different self-organized systems and interpret the experimental data. This paper is aimed at obtaining a quantitative assessment of scale invariance, self-organization in theoretical and practical aspects of computer calculations.

An important class of fractal objects forms sets that describe the geometry of percolation. Percolation theory or infiltration theory is a mathematical theory used in physics, chemistry and other fields of science to describe the emergence of connected arrays in random environments consisting of individual elements. Percolation is a critical process [8]; i.e. it assumes the existence of a certain threshold, below which the liquid propagation is limited to a finite region of the medium. Close to the critical threshold, percolation occurs along a fractal set, the geometry of which is determined exclusively by the laws of criticality. The criticality condition makes the geometric characteristics of the fractal independent of the microscopic properties of the medium. This phenomenon can be interpreted as universality.

This paper introduces a method based on theoretical approaches in the understanding of the most common behavioral patterns of complex nonlinear dynamic systems forming the states of self-organization. Article [9-12] and review [13], introduce a number of non-standard ideas for the application of fractal objects to the description of the nonlinear dynamic system revealing a self-consistent convergence to collective states. In the vicinity of the state of self-organization, the number of degrees of freedom becomes minimal. In terms of the topology of the space, this means that the fractal dimension of the space decreases due to the appearance of fractional impermeable regions that model the state of selforganization. Thus, the complexity of the nonlinear system is transferred to the complexity of the space. 
For the development of these ideas, an example of fractal manifold construction in the one-dimensional Euclidean space, based on the Cantor dust fractal is presented herein.

Pursuant to the proliferation of computing technology, an increasing number of industrial applications and an evergrowing load of research generate arrays of data from a variety of sources. Gaussian distribution is a probability distribution commonly used in statistics, signal processing, and pattern recognition. However, not all of the data to be processed is Gaussian. In this regard, methods to address this are in demand and there is a wide practice of applying the transformation of non-Gaussian data to Gaussian one. Note the classic Box Cox transformation and publications [14-15] concerned with the processing of non-Gaussian data in machine learning.

The question at issue is that possibly important information for the researcher about the physical processes that generate nonGaussian data is lost when transforming non-Gaussian data into Gaussian data and applying conventional statistical methods of data processing. The loss of universality in the description of physical processes when processing Gaussian (normal) data shows the scale of the difference.

Fractal space is of interest due to the search for a solution to the problem of processing experimental data obtained in SOC phenomena, where the strange nature of the data caused by strong mutual correlation in the short-range and long-range orders is of fundamental importance. Large-scale invariance in the phenomena of self-organized criticality derives from the exclusiveness the customary meaning of the mean as the arithmetic mean. Linear programming methods are inapplicable in the vicinity of criticality, given the requirement for scale invariance of the result. The versatility of SOC phenomena also requires versatility in the method of mathematical processing of experimental data, which would not depend on the nature of the interaction of elements in a complex nonlinear dynamic system.

The paper proposes a computational method for processing strange data directly, which allows for a quantitative comparison of the structural characteristics of big data sets without preliminary transformation into normal data.

\section{COMPUTATIONAL METHOD}

The transformed value of non-Gaussian data, which allows the quantitative comparison, must be invariant with respect to any linear transformation of the values of the original data [16]. The problem has a solution only for the ordered strange data and taking into account the vicinity in which nonlinearity reveals itself. As shown in the paper, the transformation should have a renormalization group invariance with respect to the size of the vicinity in which a quantitative comparison of the manifestations of nonlinearity takes place.

The key step in the article is the algorithm for constructing a fractal manifold - a new mathematical object in the simplest case of one-dimensional space. The idea of a fractal manifold was expressed in the scientific work [9].
The Cantor dust fractal or a geometric progression with an arbitrary value $0<q<1$ (in the classical Cantor set fractal $q=2 / 3$ ) has the symbolic form:

$$
F \sim 1-(1-q)-(1-q) q^{2}-(1-q) q^{3}-(1-q) q^{4}-\cdots
$$

The following method is proposed for a fractal manifold construction: the fractal manifold for $n=5$ of an arbitrary set of five ordered numbers $a_{i}$ has the form:

$$
\begin{aligned}
\widetilde{a_{0}^{R}}(a, 5)= & a_{0}-(1-q) a_{1}-(1-q) q a_{2} \\
& -(1-q) q^{2} a_{3}-(1-q) q^{3} a_{4} \\
& -(1-q) q^{4} a_{0}-(1-q) q^{5} a_{1} \\
& -(1-q) q^{6} a_{2}-\cdots \\
\widetilde{a_{1}^{R}}(a, 5)= & a_{1}-(1-q) a_{2}-(1-q) q a_{3} \\
& -(1-q) q^{2} a_{4}-(1-q) q^{3} a_{0} \\
& -(1-q) q^{4} a_{1}-(1-q) q^{5} a_{2} \\
& -(1-q) q^{6} a_{3}-\cdots \\
\widetilde{a_{0}^{L}}(a, 5)= & a_{0}-(1-q) a_{4}-(1-q) q a_{3} \\
& -(1-q) q^{2} a_{2}-(1-q) q^{3} a_{1} \\
& -(1-q) q^{4} a_{0}-(1-q) q^{5} a_{4} \\
& -(1-q) q^{6} a_{3}-\cdots \\
& a_{1}-(1-q) a_{0}-(1-q) q a_{4} \\
& -(1-q) q^{2} a_{3}-(1-q) q^{3} a_{2} \\
& -(1-q) q^{4} a_{1}-(1-q) q^{5} a_{0} \\
& -(1-q) q^{6} a_{4}-\cdots
\end{aligned}
$$

With each fractal cycle $m$, where $m \rightarrow \infty$, a new value $a_{i}$ appears from the sample of non-Gaussian data $n$ and then along the closed contour. Left and right directions of the contour are distinguished. Infinitely decreasing geometric progressions with different denominators are distinguished at a common factor $(1-q) a_{i}$.

In general:

$$
\widetilde{a_{l}^{R}}(a, n)=a_{i}-\frac{1-q}{1-q^{n+1}}\left[\sum_{k=1}^{n}\left(q^{k} a_{\bmod (k+1+i, n+1)}\right)\right]
$$

Similarly, for $\widetilde{a_{l}^{L}}(a, n)$, the following is obtained:

$$
\widetilde{a_{l}^{L}}(a, n)=a_{i}-\frac{1-q}{1-q^{n+1}}\left[\sum_{k=1}^{n}\left(q^{n-k} a_{\bmod (k+i, n+1)}\right)\right]
$$

The sets $\left\{\widetilde{a_{l}^{R}}(a, n)-\widetilde{a_{l}^{L}}(a, n)\right\}$ and $\left\{\widetilde{a_{l}^{R}}(a, n)+\widetilde{a_{l}^{L}}(a, n)\right\}$ form fractal manifolds, which are the first constructed example of fractal manifold. The expression for the signal-to-noise ratio $(S N R)$ is as follows:

$$
\operatorname{SNR}(a, n)=\frac{S(a, n)}{N(a, n)}=\frac{\sum_{i=0}^{n}\left(\widetilde{a_{l}^{R}}(a, n)-\widetilde{a_{l}^{L}}(a, n)\right)^{2}}{\sum_{i=0}^{n}\left(\widetilde{a_{l}^{R}}(a, n)+\widetilde{a_{l}^{L}}(a, n)\right)^{2}}
$$

The scale-invariant $S N R$ has no analogue in the spaces of integer dimension or carries the obvious meaning for the scale invariance of random numbers. The essence of the SNR in this simplest case of a one-dimensional space is similar to a new geometric characteristic of a fractal, called the connectivity 
index and introduced to describe the topology of a fractal set [9].

The main result hereof and the confirmation of the proposed constructions ((3), (4)), on which the authors rely in the development of the fractal manifold method, is the invariance of the formula (5) for the set of values of the Gauss function of the granularity $n$ for a sufficiently large number of partitions $n$. The result obtained makes it possible to note the emergence of a new mathematical criterion for Gaussian data: the data is Gaussian if the form (5) is invariant from the granularity $n$ for a sufficiently large number of partitions $n$. The discovered property of the Gauss function is independent of the method of fractal manifold construction and serves only as a justification for the chosen method of construction.

The set of values taken by the Bessel functions satisfy the proposed criterion for Gaussian data. The real data contain both random effects and collective effects caused by crosscorrelation. Gaussian noise increases the fractal dimension of the data, if not filtered using an approximation. The approximation of the data by a finite series of the Bessel functions (approximately three to seven terms of the series) leaves only the contribution of random variations. The approximation of the data by the Fourier finite series followed by the $S N R$ transformation makes it possible to identify the self-organized criticality effect.

When modeling the non-Gaussian data by the half-wave $\mathrm{a}_{i}=\sin \left(\pi \frac{i}{n}\right)$, used in calculations with the preliminary approximation of the data by the finite Fourier series for sufficiently large values of $n$, the expression for the $S N R$ has the form:

$$
\begin{aligned}
& S(n, q) \approx \frac{(1-q)^{4}(1+q)^{2}}{n-3} 2 \pi^{2}(1+4 q+\cdots) \\
& N(n, q) \approx \frac{(1-q)^{2}(1+q)^{2}}{(n-3)^{2}} 2 \pi^{2}(1+4 q+\cdots)
\end{aligned}
$$

and

$$
\operatorname{SNR}(n, q)=(1-q(n))^{2}(n-3)
$$

Require the fulfillment of the invariance condition $\operatorname{SNR}(n, q)$, which makes the strange data closer to the Gaussian ones:

$$
\frac{d}{d n} \operatorname{SNR}(n, q(n))=0
$$

The solution of the differential equation has the form:

$$
q(n)=1-\sqrt{\frac{\mu}{n-3}}
$$

The choice of the constant $\mu$ determines the scale of the SNR.

For large values of $n$, the asymptotics of the fractal manifolds' length parameters in the half-wave model has the form:

$$
l^{S} \sim n^{-\frac{3}{2}} \text { and } l^{N} \sim n^{-\frac{3}{2}}
$$

The Hausdorff fractal dimension according to Kolmogorov [17] for the fractal manifolds, constructed taking into account the direction of traversal of a closed contour of $n$ numbers, is equal to:

$$
D=-\lim _{n \rightarrow \infty}\left[\frac{\ln (n)}{\ln (l)}\right]=\frac{2}{3}
$$

The requirement of method's invariance with respect to any linear transformations of the initial data follows from the definition of fractal dimension (12). Thus, the set of values, taken by the trigonometric functions like sine and cosine, form a fractal manifold of dimension $D=2 / 3$.

The average for the Gaussian numbers:

$$
\lim _{n \rightarrow \infty}\left[\frac{1}{n} \sum_{i=0}^{n} \sin \left(\pi \frac{i}{n}\right)\right]=\frac{2}{\pi} \approx 0.64
$$

differs from the average according to Kolmogorov for $D=$ $2 / 3$.

$$
\lim _{n \rightarrow \infty}\left[\frac{1}{n} \sum_{i=0}^{n}\left(\sin \left(\pi \frac{i}{n}\right)\right)^{D}\right]^{1 / D} \approx 0.60
$$

A half-wave in the degree $p$, for integer $p$ greater than one, generates the fractal manifold of the dimension $D=2 / 5$, which is the smallest of the discovered dimensions of fractal manifolds.

An example of the fractal manifold method, the calculations are given for binomial coefficients close to the Gaussian set, normalized by the asymptotics:

$$
a_{i}=2^{-n} \sqrt{\frac{\pi n}{2}}\left[\frac{n !}{i !(n-i) !}\right]
$$

For sufficiently large values of $\mathrm{n}$, the expression of the $S N R$ is:

$$
\begin{aligned}
& S(n, q) \approx 3 \pi \frac{(1-q)^{4}}{\sqrt{2 n+1}}(1+6 q+\cdots) \\
& N(n, q) \approx 9 \pi \frac{(1-q)^{2}(1+q)^{2}}{(2 n+1)^{3 / 2}}(1+6 q+\cdots) \\
& \operatorname{SNR}(n, q)=\frac{(1-q(n))^{2}}{(1+q(n))^{2}} \frac{(2 n+1)}{3}
\end{aligned}
$$

The renormalization-group equation for $q(n)$ has the form:

$$
q(n)=\frac{\sqrt{2 n+1}-\sqrt{3 \mu}}{\sqrt{2 n+1}+\sqrt{3 \mu}}
$$

The fractal dimension for the normalized binomial coefficients is $D=4 / 5$.

The choice of the mean for the non-Gaussian data as for the Gaussian numbers, which is often made in calculations, is not unequivocal [16]. Not only the mean value itself but also the type of the mean value calculation formula is determined by the strange data. The fractal manifold method makes it possible to determine more accurately such a well-known characteristic of the structure as the average value, using the smaller scale $l \sim n^{-\frac{3}{2}}$, compared to the Euclidean scale $l^{E} \sim n^{-1}$ as an instrument and to identify qualitatively the new structural characteristic the degree of cross-correlation of the data or the degree of collective data state determined by the $S N R$.

The peculiarity of the discovered property lies in the fact that not all singularities of continuously differentiable functions are determined by an infinitely small neighborhood. The effect of cross-correlation is manifested in the short-range and longrange orders at the "microlevel" $\left(l \sim n^{-\frac{3}{2}}\right)$. 
Thus, the emergence of a dependence of the $S N R$ on the number of samples $n$ for the non-Gaussian (strange) data is explained by the presence of cross-correlation of the strange data. The introduction of the parameter $q$ from the Cantor dust fractal and the application of the renormalization-group invariance method with respect to the $S N R$ makes it possible to proceed with the traditional analysis of the Gaussian data the degree of data correlation in the $S N R$ definition (5).

Preliminary calculations are performed for $q=0$ by the formulas (24)-(26). At the preliminary stage of calculations, when comparing different sets of the ordered data, the critical sizes of the descriptors $n_{k r 1}, n_{k r 2}$ are obtained, which provide the maximum collective states in the data sets. Then the value $\mu=\left[\min \left(n_{k r 1}, n_{k r 2}\right)-3\right]$ in the formula (10) is taken and the value $\operatorname{SNR}\left(\max \left(n_{k r 1}, n_{k r 2}\right)\right)$ is calculated more accurately taking into account the invariance (20)-(23) of $q$. A comparison of the $S N R$ values of different data sets is correct in the calculation performed on a single scale $\mu$. The peaks of the $\operatorname{SNR}\left(x_{i}, n\right)$ characterize the presence of a structure in the data of the variable $x$ and delineate the vicinity of the collective state. The concept of a critical or collective state is characteristic of the strange kinetics approach, denoting a cluster of degrees of freedom that have a strong correlation [8, $9 ; 18-21]]$.

The approximation parameters of the finite Fourier series and the size of the descriptor $n$ when the ordered data are passed with unit step are determined by the conditions of the maximum of the objective function -the maximum collective state in the system .

In matrix form, the renormalization-invariant formulas for the $S N R$ are as follows:

$$
\begin{gathered}
\operatorname{SNR}(a, n)=\frac{(a \times S a)}{(a \times N a)} \\
S=-(\operatorname{matrix}(n+1, n+1, f)-\operatorname{matrix}(n+1, n+ \\
\left.1, f)^{T}\right)^{2} \\
N=[2 \text { identity }(n+1)-(\operatorname{matrix}(n+1, n+1, f)+ \\
\left.\left.\operatorname{matrix}(n+1, n+1, f)^{T}\right)\right]^{2}
\end{gathered}
$$

where

$$
f(i, j)=\frac{1-q}{1-q^{n+1}} q^{\bmod (j-i+n, n+1)}
$$

The formulas (20)-(23) are equivalent to the formulas (3)-(5) and allow for the designing of an algorithm. The formulas are the practical aspect in the solution for the problem of scale invariance and self-organized criticality, announced in the article's introduction.

In calculations, based on $K=n / 2+1$ unique ordered spectrum data, a symmetric closed-loop vector is constructed:

$$
a=\left(a_{0}, a_{1}, a_{2}, \cdots, a_{K-1}, a_{K}, a_{K-1}, \cdots, a_{2}, a_{1}\right)
$$

For $q=0$, taking into account the symmetry of the matrices $S$ and $N$, the formulas for the $S N R$ (20)-(23) acquire a usable form for processing big data:

$$
\begin{aligned}
S / 2=a_{0} & \left(a_{0}-a_{2}\right)+a_{1}\left(a_{1}-a_{3}\right) \\
& +\sum_{i=2}^{K-2} a_{i}\left(-a_{i-2}+2 a_{i}-a_{i-2}\right) \\
& +a_{K-1}\left(-a_{K-3}+a_{K-1}\right) \\
& +a_{K}\left(-a_{K-2}+a_{K}\right) \\
N / 2=a_{0}\left(3 a_{0}-\right. & \left.4 a_{1}+a_{2}\right) \\
& +a_{1}\left(-4 a_{0}+7 a_{1}-4 a_{2}+a_{3}\right) \\
& +\sum_{i=2}^{K-2} a_{i}\left(a_{i-2}-4 a_{i-1}+6 a_{i}\right. \\
& \left.-4 a_{i+1}+a_{i+2}\right) \\
& +a_{K-1}\left(a_{K-3}-4 a_{K-2}+7 a_{K-1}\right. \\
& \left.-4 a_{K}\right) \\
& +a_{K}\left(a_{K-2}-4 a_{K-1}+3 a_{K}\right)
\end{aligned}
$$

As already noted, a correct comparison of the structural SNR characteristics of different data sets should be carried out on a single scale $\mu$ with allowance for $q$ invariance (20)-(23).

The method is used for large sets of data obtained in good resolution, which makes it possible to increase the scale of the comparison $\mu$ with the preservation of the invariance.

\section{CONCLUSIONS}

The fractal manifold method makes it possible to process nonGaussian data without prior transformation into the usual Gaussian data, which gives the researchers an option of expanding the range of problems to be solved and allows for obtaining more information about the processes that generate non-Gaussian data arrays. The proposed method is in demand when the cross-correlation of data in the array is significant, reflecting such processes as avalanches, chain reactions and collective effects. It becomes possible to quantify SOC events.

The solution to the problem of processing directly nonGaussian data is based on a new mathematical construction fractal manifold (formulas (3)-(5)). The success in solving the problem under consideration is confirmed by the revealed property for the set of values taken by the Gauss function, namely, in the invariance of the form (5) of the granularity $n$ for sufficiently large values of $n$. The Gauss and Bessel functions are widely used in various fields of knowledge and the discovery of a new mathematical property in them in the proposed research is an independent result. The revealed Gauss function property makes it possible to propose a new criterion for Gaussian data. For example, it is shown that the binomial distribution is a fractal manifold with dimension $D=4 / 5$ and it does not satisfy the criterion for Gaussian data, and the set of values taken by the Bessel functions is Gaussian.

The fractal manifold method makes it possible to determine more accurately the average value due to the smaller scale compared to the Euclidean scale in a one-dimensional case.

There is a fundamental possibility of quantitative comparison of SOC effects, manifested in different fields of knowledge. In accordance with the universality of the topological approach, it becomes a reality, for example, a quantitative comparison of the collective effect in sociology and cosmophysics. 
The construction of a fractal manifold for a closed contour is achieved using simple rules (formulas (3)-(5)). At the same time, the indicated formulas are instrumental only in analytical calculations and can not be applied to processing large scale arrays of data. In the proposed study, it is recommended to use the formulas (20)-(23), which allow for processing large scale arrays of data. The formulas (20)-(23) are obtained by proceeding from the condition that the results are identical with analytical calculations.

The proposed fractal manifold method for processing nonGaussian data is an approximate approach; the main factor of approximation is the preliminary approximation of nonGaussian data by a finite Fourier series. Fourier approximation reduces the effect of random Gaussian values. Further research is required on the method of estimation of the error in nonGaussian data processing when traditional statistical approaches are not applicable. In determining the reliability of non-Gaussian data processing, the fractal dimension of the approximating functions is of great importance. Gaussian noise increases the fractal dimension if not filtered. The smaller the fractal dimension of the approximating functions, the more accurately the dimension of the criticality is determined. A limitation in the proposed approach is the consideration of only one-dimensional arrays of non-Gaussian data.

The prospective $[3,13]$ to the concept of self-organized criticality, which is shared by many researchers as having a paradigmatic meaning is noted in the introduction section. In this regard, there is a demand for a method of setting the problem that would correspond to the new paradigm in physics - what is to be determined? The present study is aimed at the identification of some marks (features) in the future formulation of the problem.

\section{REFERENCES}

[1] Bak P, Tang C, Wiesenfeld K. Self-organized criticality: an explanation of 1/f noise, 1987. Phys. Rev. Lett.;59:381.

[2] Bak P, Tang C, Wiesenfeld K. Self-organized criticality. Physical review A. 1988 Jul 1;38(1):364.

[3] Anderson PW. More is different. Science. 2011;177(4047):393-6.

[4] Milovanov AV. Percolation models of self-organized critical phenomena. arXiv preprint arXiv:1207.5389. 2013 Jul 23.

[5] Meisel C, Storch A, Hallmeyer-Elgner S, Bullmore E, Gross T. Failure of adaptive self-organized criticality during epileptic seizure attacks. PLoS Comput Biol. 2012 Jan 5;8(1):e1002312.

[6] Hoffmann H, Payton DW. Optimization by selforganized criticality. Scientific reports. 2018 Feb $5 ; 8(1): 1-9$

[7] Kadanoff LP. Fractals: Where's the Physics?. Phys Today. 1986;39(2): 6.

[8] Isichenko MB. Percolation, statistical topography, and transport in random media. Reviews of modern physics. 1992 Oct 1;64(4):961.
[9] Milovanov AV. Topological proof for the AlexanderOrbach conjecture. Physical Review E. 1997 Sep $1 ; 56(3): 2437-2446$.

[10] Milovanov AV, Zimbardo G. Percolation in signsymmetric random fields: topological aspects and numerical modeling. Physical Review E. 2000 Jul $1 ; 62(1): 250$.

[11] Milovanov AV, Rasmussen JJ. Critical conducting networks in disordered solids: ac universality from topological arguments. Physical Review B. 2001 Nov $13 ; 64(21): 212203$.

[12] Milovanov AV, Rasmussen JJ. Fracton pairing mechanism for unconventional superconductors: Selfassembling organic polymers and copper-oxide compounds. Physical Review B. 2002 Oct 7;66(13):134505.

[13] Zelenyi LM, Milovanov AV. Advances in the physical sciences, Fractal topology, and strange kinetics: from percolation theory to the problems of cosmic electrodynamics. Phys. Usp. 2004; 47: 749-788.

[14] Neal RM. Priors for infinite networks. InBayesian Learning for Neural Networks 1996 (pp. 29-53). Springer, New York, NY.

[15] Jacot A, Gabriel F, Hongler C. Neural tangent kernel: Convergence and generalization in neural networks. InAdvances in neural information processing systems 2018 (pp. 8571-8580).

[16] Orlov AI. Applied Statistics. Moscow: Exam. 2006.

[17] Kolmogorov AN. New metric invariant of transitive dynamical systems and automorphisms of Lebesgue spaces. Reports of the Academy of Sciences of the USSR, 1958;5: 861-864.

[18] Yemelyanov VA, Yemelyanova NY, Shved EV, Nedelkin AA, Fatkulin AR. Modeling of the Multilayer Perceptrons for Image Recognition of the Steel Microstructures. In2020 IEEE Conference of Russian Young Researchers in Electrical and Electronic Engineering (EIConRus) 2020 Jan 27 (pp. 952-955). IEEE.

[19] Rahman PA, Panchenko AA, Safarov AM. Using neural networks for prediction of air pollution index in industrial city. InIOP Conference Series: Earth and Environmental Science 2017 Oct (Vol. 87, No. 4, p. 042016). IOP Publishing.

[20] Sychev Y, Abramovich B, Prokhorova V. The assesement of the shunt active filter efficiency under varied power supply source and load parameters. International Journal of Electrical \& Computer Engineering (2088-8708). 2020 Dec 1;10(6):5621-5630.

[21] Movchan IB, Yakovleva AA. Refined assessment of seismic microzonation with a priori data optimisation. Записки Горного института. 2019;236:133-141. 